TRANSACTIONS OF THE

AMERICAN MATHEMATICAL SOCIETY

Volume 365, Number 6, June 2013, Pages 3019-3039

S 0002-9947(2012)05687-X

Article electronically published on December 13, 2012

\title{
CONNES EMBEDDINGS AND VON NEUMANN REGULAR CLOSURES OF AMENABLE GROUP ALGEBRAS
}

\author{
GÁBOR ELEK
}

\begin{abstract}
The analytic von Neumann regular closure $R(\Gamma)$ of a complex group algebra $\mathbb{C} \Gamma$ was introduced by Linnell and Schick. This ring is the smallest *-regular subring in the algebra of affiliated operators $U(\Gamma)$ containing $\mathbb{C} \Gamma$. We prove that all the algebraic von Neumann regular closures corresponding to sofic representations of an amenable group are isomorphic to $R(\Gamma)$. This result can be viewed as a structural generalization of Lück's approximation theorem.

The main tool of the proof which might be of independent interest is that an amenable group algebra $K \Gamma$ over any field $K$ can be embedded to the rank completion of an ultramatricial algebra.
\end{abstract}

\section{INTRODUCTION}

1.1. Regular rank rings. In this paper all rings are considered unital. Regular rings were introduced by John von Neumann; these are the rings where any principal right ideal is generated by an idempotent (see [9]). A *-regular ring $\mathcal{R}$ is a ring with involution and $a^{*} a=0$ implies that $a=0$. In a $*$-regular ring any principal right ideal is generated by a unique projection [11. A *-regular ring $\mathcal{R}$ is proper if $\sum_{i=1}^{n} a_{i} a_{i}^{*}=0$ implies that all of the $a_{i}$ 's are equal to 0 . Note that $\operatorname{Mat}_{d \times d}(\mathcal{R})$ is regular if and only if $\mathcal{R}$ is regular, nevertheless for a $*$-regular $\operatorname{ring} \mathcal{R} \operatorname{Mat}_{d \times d}(\mathcal{R})$ is

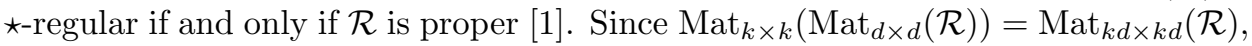
$\mathcal{R}$ is proper if and only if all the matrix rings over $\mathcal{R}$ are proper.

A rank function on a regular ring $\mathcal{R}$ is function rk $\mathcal{R} \rightarrow \mathbb{R}$ satisfying the following conditions:

(1) $0 \leq \operatorname{rk}(a) \leq 1$.

(2) $\operatorname{rk}(a)=0$ if and only if $a=0$.

(3) $\operatorname{rk}(a+b) \leq \operatorname{rk}(a)+\operatorname{rk}(b)$.

(4) $\operatorname{rk}(a b) \leq \operatorname{rk}(a), \operatorname{rk}(b)$.

(5) If $e, f$ are orthogonal idempotents, then $\operatorname{rk}(e+f)=\operatorname{rk}(e)+\operatorname{rk}(f)$.

The most important examples of regular rank rings are matrix rings over division rings $D$. Recall that for $A \in \operatorname{Mat}_{d \times d}(D), \operatorname{rk}(A)=\frac{\operatorname{rank}(A)}{d}$, where rank is the usual matrix rank. In this case, the values of the rank are always rational. The rank defines a metric on the regular ring by $d(x, y)=\operatorname{rk}(x-y)$. The completion of this metric is a regular rank ring as well. Note that for the completion of ultramatricial algebras (see Section 4) the values of the rank can be any real number in between

Received by the editors July 6, 2010 and, in revised form, March 15, 2011, May 3, 2011, and August 21, 2011.

2010 Mathematics Subject Classification. Primary 16S34, 22D25.

This research was sponsored by OTKA Grant No. 69062.

(C)2012 American Mathematical Society Reverts to public domain 28 years from publication 
zero and one [9]. Let $\mathcal{N}$ be a finite tracial von Neumann algebra; then, its Ore localization with respect to its non-zero divisiors $U(\mathcal{N})$ is a $\star$-regular ring. The elements of this ring are called affiliated operators (see [19]). The rank of an affiliated operator is the trace of the idempotent that generates the right ideal generated by the operator. Note that if $A \in U(\mathcal{N})$, then

$$
\operatorname{rk}(A)=1-\lim _{\lambda \rightarrow 0} \operatorname{tr}_{\mathcal{N}}\left(E_{\lambda}\right)
$$

where $\int_{0}^{\infty} \lambda d E \lambda$ is the spectral decomposition of the unbounded operator $A^{*} A$. This shows that if $i: \mathcal{N} \rightarrow \mathcal{M}$ is a trace-preserving homomorphism between finite tracial von Neumann algebras, then its Ore localization $\tilde{i}: U(\mathcal{N}) \rightarrow U(\mathcal{M})$ is a rank preserving $\star$-homomorphism. By [21, Lemma 2.2], $U(\mathcal{N})$ is just the rank completion of $\mathcal{N}$.

Note that $U(\mathcal{N})$ is always proper (see Section 3).

If $\mathcal{R}$ is a regular rank ring, then there is a unique natural extension of the rank to a matrix rank of $\operatorname{Mat}_{k \times k}(\mathcal{R})$ [10. Note that a matrix rank rkm has the same property as the rank rk except that $0 \leq \operatorname{rkm}(M) \leq k$.

1.2. The Connes embedding problem. Let $\mu=\left\{d_{1}<d_{2}<\ldots\right\}$ be an infinite sequence of positive integers. Then one can consider the ultraproduct of the matrix algebras $\left\{\operatorname{Mat}_{d_{i} \times d_{i}}(\mathbb{C})\right\}_{i=1}^{\infty}$ as tracial algebras in the following way (see [18]).

Let $\omega$ be a non-principal ultrafilter on the natural numbers and let $\lim _{\omega}$ be the corresponding ultralimit. First, consider the algebra of bounded elements

$$
\mathcal{B}=\left\{\left(a_{1}, a_{2}, \ldots\right) \in \prod_{i=1}^{\infty} \operatorname{Mat}_{d_{i} \times d_{i}}(\mathbb{C}) \mid \sup \left\|a_{i}\right\|<\infty\right\}
$$

Now let $\mathcal{I} \triangleleft \mathcal{B}$ be the ideal of elements $\left\{a_{i}\right\}_{i=1}^{\infty}$ such that $\lim _{\omega} \frac{\operatorname{tr}\left(a_{n}^{*} a_{n}\right)}{d_{n}}=0$. Then $\mathcal{B} / \mathcal{I}=\mathcal{M}_{\mu}$ is a type $I I_{1}$-von Neumann factor with trace defined by

$$
\operatorname{Tr}_{\omega}\left[\left\{a_{i}\right\}_{i=1}^{\infty}\right]=\lim _{\omega} \frac{\operatorname{tr}\left(a_{n}\right)}{d_{n}} .
$$

The following conjecture is generally referred to as the Connes embedding problem: Is it true that a type- $I I_{1}$ von Neumann algebra with a separable predual has a trace-preserving embedding to some $\mathcal{M}_{\mu}$ ? See the survey of Pestov [18] for further details.

There is a purely algebraic version of the Connes embedding problem first considered in [7]. Namely, we can consider the ultraproduct of the matrix rings $\left\{\operatorname{Mat}_{d_{i} \times d_{i}}(\mathbb{C})\right\}$ as rank algebras.

Definition 1.1. Let $\mathcal{J} \triangleleft \prod_{i=1}^{\infty} \operatorname{Mat}_{d_{i} \times d_{i}}(\mathbb{C})$ be the following ideal,

$$
\mathcal{J}=\left\{\left\{a_{i}\right\}_{i=1}^{\infty} \mid \lim _{\omega} \frac{\operatorname{rank}\left(a_{i}\right)}{d_{i}}=0\right\} .
$$

Then $\prod_{i=1}^{\infty} \operatorname{Mat}_{d_{i} \times d_{i}}(\mathbb{C}) / \mathcal{J}=\mathcal{M}_{\mu}^{\text {alg }}$ is the ultraproduct of the matrix rings $\left\{\operatorname{Mat}_{d_{i} \times d_{i}}(\mathbb{C})\right\}$.

$\mathcal{M}_{\mu}^{a l g}$ is a simple complete $\star$-regular rank ring (see [7, Proposition 3.3]). It is important to note that $\mathcal{M}_{\mu}^{\text {alg }}$ depends on the choice of the ultrafilter. One can ask of course, whether any countable dimensional regular rank ring embeds to some $\mathcal{M}_{\mu}^{a l g}$. 
1.3. Lück's approximation theorem. Let $\Gamma$ be a finitely generated residually finite group and let $\Gamma=N_{0} \supset N_{1} \supset N_{2}, \ldots, \bigcap_{k=1}^{\infty} N_{k}=\{1\}$ be finite index normal subgroups. Let $\Delta \in \operatorname{Mat}_{d \times d}(\mathbb{Z} \Gamma)$ be a $d \times d$-matrix over the integer group algebra $\mathbb{Z} \Gamma$. Denote by $\mathcal{N}(\Gamma)$ the von Neumann algebra of $\Gamma$. Note that $\Delta$ acts on $l^{2}(\Gamma)^{d}$ as a bounded operator. Then one can define $\operatorname{dim}_{\Gamma} \operatorname{Ker}(\Delta)$ the von Neumann dimension of the kernel of $\Delta$ by

$$
\operatorname{dim}_{\Gamma} \operatorname{Ker}(\Delta):=\sum_{i=1}^{d}\left\langle P_{K e r(\Delta)} e_{i}, e_{i}\right\rangle,
$$

where $P_{\operatorname{Ker}(\Delta)}$ is the orthogonal projection onto $\operatorname{Ker}(\Delta)$ and $e_{i}$ is the unit vector supported on the unit element of the $i$-th component of $l^{2}(\Gamma)^{d}$. Let $\pi_{k}: \mathbb{C} \Gamma \rightarrow$ $\mathbb{C}\left(\Gamma \backslash N_{k}\right)$ be the natural projections. That is $\pi_{k}(\Delta) \in \operatorname{Mat}_{d \times d}\left(\mathbb{C}\left(\Gamma \backslash N_{k}\right)\right)$ is a finite dimensional linear transformation. According to Lück's approximation theorem (see [15])

$$
\lim _{k \rightarrow \infty} \frac{\operatorname{dim}_{\mathbb{C}} \operatorname{Ker}\left(\pi_{k}(\Delta)\right)}{\left|\Gamma: N_{k}\right|}=\operatorname{dim}_{\Gamma} \operatorname{Ker}(\Delta) .
$$

Note that (2) holds even if the coefficients are algebraic numbers [3]. It is conjectured that (2) holds for any $\Delta \in \operatorname{Mat}_{d \times d}(\mathbb{C} \Gamma)$ as well. The conjecture was confirmed for amenable groups $\Gamma$ in [8] (see also the remark at the end of Section 8).

1.4. Regular closures. Linnell and Schick 13] proved the following theorem (see Section 3 . Let $\mathcal{R}$ be a proper $\star$-regular ring. Then for any subset $T \subseteq \mathcal{R}$ there exists a smallest $\star$-regular subring containing $T$. We call this $\operatorname{ring} R(T, \mathcal{R})$ the regular closure of $T$ in $\mathcal{R}$. Let $\Gamma$ be a countable group; then one can consider the natural embedding of its complex group algebra into its von Neumann algebra $\mathbb{C}(\Gamma) \rightarrow \mathcal{N}(\Gamma)$. Let $\mathcal{U}(\Gamma)$ be the Ore localization of $\mathcal{N}(\Gamma)$. Then $\mathcal{U}(\Gamma)$ is a proper *-regular ring (see [2]). Therefore one can consider the analytic regular closure $R(\mathbb{C}(\Gamma), \mathcal{U}(\Gamma))=R(\Gamma)$.

Now let $\Gamma=N_{0} \triangleright N_{1} \triangleright \ldots, \bigcap_{i=1}^{\infty} N_{i}=\{1\}$ be finite index normal subgroups of a residually finite group. Let $\pi_{i}: \mathbb{C} \Gamma \rightarrow \mathbb{C}\left(\Gamma / N_{i}\right)$ be the natural projection as in the previous subsection and let $s_{i}: \mathbb{C}\left(\Gamma / N_{i}\right) \rightarrow \operatorname{Mat}_{\Gamma / N_{i} \times \Gamma / N_{i}}(\mathbb{C})$ be the natural representations by convolutions. Define $r_{i}=s_{i} \circ \pi_{i}: \mathbb{C} \Gamma \rightarrow \operatorname{Mat}_{\Gamma / N_{i} \times \Gamma / N_{i}}(\mathbb{C})$. Then we have an injective (see [7, Theorem 1]) $\star$-homomorphism $r: \mathbb{C} \Gamma \rightarrow \mathcal{M}_{\mu}^{\text {alg }}$, where $\mu=\left\{\left|\Gamma / N_{1}\right|,\left|\Gamma / N_{2}\right|, \ldots\right\}$. Therefore we can consider the algebraic regular closure $R\left(\mathbb{C} \Gamma, \mathcal{M}_{\mu}^{a l g}\right)$ for any normal chain of a residually finite group. The main result of this paper is the following theorem.

Theorem 1. Let $\Gamma$ be a finitely generated amenable group. Then there is a rank preserving $\star$-homomorphism

$$
j: R(\Gamma) \rightarrow R\left(\mathbb{C} \Gamma, \mathcal{M}_{\mu}^{\text {alg }}\right)
$$

which is the identity map restricted on $\mathbb{C} \Gamma$.

This theorem can be viewed as a structural generalization of Lück's approximation theorem for amenable groups. Indeed, let $\Delta \in \operatorname{Mat}_{d \times d}(\mathbb{C} \Gamma)$. Also, let $\operatorname{rkm}_{1}(\Delta)$ be the matrix rank of $\Delta$ in the ring $\operatorname{Mat}_{d \times d}(U(\Gamma))$, respectively; let $\operatorname{rkm}_{2}(\Delta)$ be the matrix rank of $\Delta$ in $\operatorname{Mat}_{d \times d}\left(\mathcal{M}_{\mu}^{a l g}\right)$. By Theorem 1,

$$
\operatorname{rkm}_{1}(\Delta)=\operatorname{rkm}_{2}(\Delta)
$$


since both sides are equal to the matrix rank of $\Delta$ in $\operatorname{Mat}_{d \times d}(R(\Gamma))$. Observe that $\operatorname{rkm}_{1}(\Delta)=d-\operatorname{dim}_{\Gamma} \operatorname{ker}(\Delta)$. On the other hand,

$$
\operatorname{rkm}_{2}(\Delta)=d-\lim _{\omega} \frac{\operatorname{dim}_{\mathbb{C}} \operatorname{Ker}\left(\pi_{k}(\Delta)\right)}{\left|\Gamma: N_{k}\right|} .
$$

Since, by (3), $\operatorname{rkm}_{2}(\Delta)$ does not depend on the choice of the ultrafilter, then $\lim _{k \rightarrow \infty} \frac{\operatorname{dim}_{\mathbb{C}} K \operatorname{er}\left(\pi_{k}(\Delta)\right)}{\left|\Gamma: N_{k}\right|}$ must exist and (2) follows.

Actually, we prove a generalization of Theorem 1, where we consider algebraic closures associated to arbitrary sofic representations (see Section 7) of the group $\Gamma$. We shall also prove the following theorem.

Theorem 2. For any finitely generated amenable group and coefficient field $K$, there exists a rank preserving embedding of the group algebra $K \Gamma$ into the rank completion of an ultramatricial algebra.

\section{The Strategy of the PRoof}

In our paper we consider four complete proper regular $*$-rings: $\overline{\mathcal{A}_{\phi}}$ (this will be defined in Section 4), $U(\Gamma), \mathcal{M}_{\mu}^{a l g}$ and $U\left(\mathcal{M}_{\mu}\right)$. Also, we will define seven rank preserving embeddings:

(1) $f_{1}: \mathbb{C} \Gamma \rightarrow \mathcal{M}_{\mu}^{\text {alg }}$.

(2) $f_{2}: \mathbb{C} \Gamma \rightarrow U(\Gamma)$.

(3) $f_{3}: \mathbb{C} \Gamma \rightarrow U\left(\mathcal{M}_{\mu}\right)$.

(4) $f_{4}: \mathbb{C} \Gamma \rightarrow \overline{\mathcal{A}_{\phi}}$.

(5) $f_{5}: \overline{\mathcal{A}_{\phi}} \rightarrow \mathcal{M}_{\mu}^{a l g}$.

(6) $f_{6}: \overline{\mathcal{A}_{\phi}} \rightarrow U\left(\mathcal{M}_{\mu}\right)$.

(7) $f_{7}: U(\Gamma) \rightarrow U\left(\mathcal{M}_{\mu}\right)$.

In the final section, we shall prove three identities:

(1) $f_{5} \circ f_{4}=f_{1}$.

(2) $f_{6} \circ f_{4}=f_{3}$.

(3) $f_{7} \circ f_{2}=f_{3}$.

From these identities, the main theorem easily follows. Indeed, $R(\Gamma)$ is the smallest $\star$-regular ring containing $\mathbb{C} \Gamma$ in $U(\Gamma)$. The ring $R(\Gamma)$ is inside $\overline{\mathcal{A}_{\phi}}$; in fact, it is the minimal $\star$-regular ring containing $\mathbb{C} \Gamma$ in $\overline{\mathcal{A}_{\phi}}$. On the other hand, $R\left(\mathbb{C} \Gamma, \mathcal{M}_{\mu}^{\text {alg }}\right)$ is also the smallest $\star$-regular ring containing $\mathbb{C} \Gamma$ in $\overline{\mathcal{A}_{\phi}}$.

\section{VON NEUMANN REgUlar ClOSURES}

In this section, we review some results of Linnell and Schick [12, 13, about the von Neumann regular closures in proper $\star$-regular rings. The starting points of Linnell's paper are the following two observations about finite von Neumann algebras already mentioned in the introduction.

(1) $U(\mathcal{N})$ is a $\star$-regular ring, that is, any right ideal is generated by a single projection.

(2) If $\alpha, \beta \in U(\mathcal{N})$ and $\alpha \alpha^{*}+\beta \beta^{*}=0$, then $\alpha=\beta=0$.

Although Linnell and Schick consider only group von Neumann algebras, all they used are the two properties above. The following result is a strengthening of the second observation. 
Proposition 3.1 ([12, Lemma 2]). If $\alpha, \beta \in U(\mathcal{N})$, then $\left(\alpha \alpha^{*}+\beta \beta^{*}\right) U(\mathcal{N}) \supseteq$ $\alpha U(\mathcal{N})$.

Using a simple induction one also has the following proposition.

Proposition 3.2 ([13, Lemma 2.5]). If $\alpha_{1}, \alpha_{2}, \ldots, \alpha_{n} \in U(\mathcal{N})$, then

$$
\sum_{i=1}^{n} \alpha_{i} \alpha_{i}^{*} U(\mathcal{N}) \supseteq \alpha_{1} U(\mathcal{N}) .
$$

This leads to the crucial proposition about the existence of the von Neumann regular closures.

Proposition 3.3 ([13, Proposition 3.1]). Let $\left\{R_{i} \mid i \in I\right\}$ be a collection of $\star$ regular subrings of $U(\mathcal{N})$. Then $\bigcap_{i \in I} R_{i}$ is also a $\star$-regular subring of $U(\mathcal{N})$.

We also need to show that the proposition above holds for $\mathcal{M}_{\mu}^{a l g}$ as well.

Proposition 3.4. Let $\left\{R_{i} \mid i \in I\right\}$ be a collection of $\star$-regular subrings of $\mathcal{M}_{\mu}^{\text {alg }}$. Then $\bigcap_{i \in I} R_{i}$ is also a -regular subring of $\mathcal{M}_{\mu}^{\text {alg }}$.

Proof. Since $\mathcal{M}_{\mu}^{\text {alg }}$ is a $\star$-regular ring, we only need to prove that if $\alpha \alpha^{*}+\beta \beta^{*}=0$ in $\mathcal{M}_{\mu}^{\text {alg }}$, then both $\alpha$ and $\beta$ are equal to 0 . Then the proof of Proposition 3.3 works without any change.

Lemma 3.1. For finite dimensional matrices $A, B \in \operatorname{Mat}_{k \times k}(\mathbb{C})$,

$$
\operatorname{rank}\left(A A^{*}+B B^{*}\right) \geq \max (\operatorname{rank}(A), \operatorname{rank}(B)) .
$$

Proof. If $\left(A A^{*}+B B^{*}\right)(v)=0$, then $A^{*}(v)=0$ and $B^{*}(v)=0$. Hence

$$
\operatorname{ker}\left(A A^{*}+B B^{*}\right) \subseteq \operatorname{ker}\left(A^{*}\right) \cap \operatorname{ker}\left(B^{*}\right) .
$$

Therefore $\operatorname{rank}\left(A A^{*}+B B^{*}\right) \geq \operatorname{rank}\left(A^{*}\right)=\operatorname{rank}(A)$ and $\operatorname{rank}\left(A A^{*}+B B^{*}\right) \geq$ $\operatorname{rank}\left(B^{*}\right)=\operatorname{rank}(B)$.

Now let $A_{n}, B_{n} \in \operatorname{Mat}_{d_{n} \times d_{n}}(\mathbb{C})$, then

$$
\lim _{\omega} \frac{\operatorname{rank}\left(A_{n} A_{n}^{*}+B_{n} B_{n}^{*}\right)}{d_{n}} \geq \lim _{\omega} \frac{\operatorname{rank}\left(A_{n}\right)}{d_{n}}
$$

and

$$
\lim _{\omega} \frac{\operatorname{rank}\left(A_{n} A_{n}^{*}+B_{n} B_{n}^{*}\right)}{d_{n}} \geq \lim _{\omega} \frac{\operatorname{rank}\left(B_{n}\right)}{d_{n}} .
$$

Hence the proposition follows.

\section{Bratteli Diagrams, ultramatricial algebras AND tilings}

Recall that a Bratteli diagram is an oriented countable graph with labeled edges and vertices such that the vertex set is partitioned into finite sets $\left\{Z_{i}\right\}_{i=1}^{\infty}$ in such a way that:

- If the starting vertex of an edge is in $Z_{i}$, then the end vertex is necessarily in $Z_{i+1}$.

- Each vertex has at least one outgoing edge.

- Each vertex $A$ has a non-negative size $S(A)$.

- Each edge (from a vertex $A$ to a vertex $B$ ) has a non-negative multiplicity $K(A, B)$ such that for each $B \in Z_{n+1}, S(B)=\sum_{A \in Z_{n}} S(A) K(A, B)$. Note that $K(A, B)=0$ if there is no edge between $A$ and $B$. 
We call a function $m: \bigcup_{n=1} Z_{n} \rightarrow[0,1]$ a harmonic function if:

- $\sum_{A \in Z_{n}} m(A)=1$ for any $n \geq 1$.

$$
m(A)=\sum_{B \in Z_{n+1}} \frac{S(A) K(A, B)}{S(B)} m(B)
$$

for any $n \geq 1$ and $A \in Z_{n}$.

4.1. Construction of the ultramatricial algebra. First of all, let $\left(\bigcup_{n=1}^{\infty} Z_{n}, m\right)$ be a Bratteli diagram with a harmonic function. We shall use the following convention in our paper. If $A$ is a set and $K$ is a field, then we will consider the matrix ring $\operatorname{Mat}_{A \times A}(K)$. Each element of $\operatorname{Mat}_{A \times A}(K)$ is regarded as a kernel map $T: A \times A \rightarrow K$, where:

- $(T+S)(p, q)=T(p, q)+S(p, q)$.

- $T S(p, q)=\sum_{r \in A} T(p, r) S(r, q)$.

- If $K=\mathbb{C}$, then $T^{*}(p, q)=\overline{T(p, q)}$.

- If $v=\sum_{p \in A} r_{p} e_{p} \in K^{A}$, then $T(v)=\sum_{q \in A}\left(\sum_{p \in A} T(q, p) r_{p}\right) e_{q}$.

For each $n \geq 0$, we regard each element of $Z_{n}$ as a finite set of size $|A|=S(A)$. For each $A \in Z_{n}$ and $B \in Z_{n+1}$, we fix $K(A, B)$ injective maps $f_{1}^{A, B}, f_{2}^{A, B}, \ldots, f_{K(A, B)}^{A, B}$ from $A$ to $B$ in such a way that

$$
\bigcup_{A \in Z_{n}} \bigcup_{i=1}^{K(A, B)} f_{i}^{A, B}(A)
$$

gives rise to a partition of $B$. Using this data, we construct an injective homomorphism

$$
E_{B}: \bigoplus_{A \in Z_{n}} \operatorname{Mat}_{A \times A}(K) \rightarrow \operatorname{Mat}_{B \times B}(K)
$$

the following way. If $\bigoplus_{A \in Z_{n}} T_{A} \in \bigoplus_{A \in Z_{n}} \operatorname{Mat}_{A \times A}(K), x \in B, x=f_{j}^{A_{i}, B}(p)$, where $p \in A_{i}$, then

$$
E_{B}\left(\bigoplus_{A \in Z_{n}} T_{A}\right)\left(e_{x}\right)=\sum_{q \in A_{i}} T(q, p) e_{f_{j}^{A_{i}, B}(q)} .
$$

Note that $E_{B}\left(T_{A_{1}} \oplus T_{A_{2}} \oplus \cdots \oplus T_{A_{i_{n}}}\right)$ is a block diagonal matrix, where exactly $K\left(A_{i}, B\right)$ of the blocks are equal to the matrix $T_{A_{i}}$.

The embeddings $E_{B}$ define injective maps

$$
\phi_{n}: \bigoplus_{A \in Z_{n}} \operatorname{Mat}_{A \times A}(K) \rightarrow \bigoplus_{B \in Z_{n+1}} \operatorname{Mat}_{B \times B}(K)
$$

by $\phi_{n}=\bigoplus_{B \in Z_{n+1}} E_{B}$. The direct limit $\lim _{\rightarrow} \phi_{n}$ is the ultramatricial algebra $\mathcal{A}_{\phi}$. Clearly, $\mathcal{A}_{\phi}$ is a regular ring ( $\star$-regular if $K=\mathbb{C}$ ).

The harmonic function defines a rank function $\operatorname{rk}_{\phi}$ on $\mathcal{A}_{\phi}$. Namely, if $T_{A} \in$ Mat $_{A \times A}, A \in Z_{n}$, then let

$$
\operatorname{rk}_{\phi}\left(\bigoplus_{A \in Z_{n}} T_{A}\right)=\sum_{A \in Z_{n}} m(A) \frac{\operatorname{rank}\left(T_{A}\right)}{|A|}
$$

where $\operatorname{rank}\left(T_{A}\right)$ is the rank of the matrix $T_{A}$. Then it is easy to see that each $\phi_{n}$ is a rank preserving $\star$-isomorphism. Therefore $\mathcal{A}_{\phi}$ is a rank regular ring. 
Now let $\Gamma$ be a finitely generated group with a symmetric generating system $S$. The Cayley graph of $\Gamma, \operatorname{Cay}(\Gamma, S)$ is defined as follows:

- $V(\operatorname{Cay}(\Gamma, S))=\Gamma$.

- $(a, b) \in E(C a y(\Gamma, S))$ if $a s=b$ for some $s \in S$.

Let $F \subset \Gamma$ be a finite set. Then $\partial F$ is the set of vertices in $F$ that are adjacent to a vertex in the complement of $F$. The isoperimetric constant of $F$ is defined as

$$
i(F):=\frac{|\partial F|}{|F|} .
$$

The group $\Gamma$ is amenable if there exists a $F \varnothing l n e r-s e q u e n c e$ in $\Gamma$, that is, a sequence of finite sets $\left\{F_{n}\right\}_{n=1}^{\infty}$ such that $\lim _{n \rightarrow \infty} i\left(F_{n}\right)=0$. If $\gamma \in \Gamma, F \subset \Gamma$, then $\gamma F$ is called an $F$-tile.

Definition 4.1 (Bratteli tiling system). A Bratteli tiling system has the following properties:

- The level set $Z_{n}$ consists of finite subsets of a group $\Gamma, F_{1}^{n}, F_{2}^{n}, \ldots, F_{i_{n}}^{n}$ and the set $E_{n}$ containing only the unit element. Also, we have $i\left(F_{j}^{n}\right) \leq \frac{1}{2^{n}}$ for all $j$ and $n$.

- For any $n \geq 2$ and $F_{j}^{n} \in Z_{n}$ we have a partition $F_{j}^{n}=\bigcup_{i=1}^{a_{n, j}} \gamma_{i} A_{i}$, where $A_{i} \in Z_{n-1}$. That is, we have tiling of $F_{j}^{n}$ with the tiles of $Z_{n-1}$.

- $K\left(F_{l}^{n-1}, F_{j}^{n}\right)$ is the number of $F_{l}^{n-1}$-tiles in the partition of $F_{j}^{n}$. Also $K\left(E_{n-1}, F_{j}^{n}\right)$ is the number of $E_{n-1}$-tiles (single vertices).

- $S\left(F_{j}^{n}\right)=\left|F_{j}^{n}\right|, S\left(E_{n}\right)=1$.

- We also suppose that $K\left(E_{n-1}, F_{j}^{n}\right) \leq \frac{1}{2^{n}}\left|F_{j}^{n}\right|$.

- We have a harmonic function $m: \bigcup_{n=1}^{\infty} Z_{n} \rightarrow[0,1]$ such that $m\left(E_{n}\right) \rightarrow 0$ as $n \rightarrow \infty$.

Our main technical tool is the following proposition.

Proposition 4.1. For any amenable group $\Gamma$ and generating system $S$ we can construct a Bratteli tiling system with the following property. For any $\epsilon>0$ and $n>0$ there exists $\delta=\delta_{\varepsilon, n}>0$ such that if $H \in \Gamma$ is a finite set and $i(H)<\delta$, then one can tile $H$ with translates of the elements $Z_{n}$ in such a way that if $A \in Z_{n}$ and $T_{A}^{H}$ is the set of points in $H$ covered by a translate of $A$, then

$$
\left|\frac{\left|T_{A}^{H}\right|}{|H|}-m(A)\right|<\epsilon .
$$

\section{Proof of Proposition 4.1}

First, let us recall the notion of $\varepsilon$-quasitilings. Let $\operatorname{Cay}(\Gamma, S)$ be the Cayley-graph of an amenable group $\Gamma$ as above. Let $H \subset \Gamma$ be a finite set and $A_{1}, A_{2}, \ldots, A_{n}$ be subsets of $H$. We say that $\left\{A_{i}\right\}_{i=1}^{n} \varepsilon$-cover $H$ if

$$
\frac{\left|\bigcup_{i=1}^{n} A_{i}\right|}{|H|}>1-\varepsilon \text {. }
$$

Also, we call $\left\{A_{i}\right\}_{i=1}^{n} \varepsilon$-disjoint if there exist mutually disjoint sets $\left\{B_{i}\right\}_{i=1}^{n}, B_{i} \subset$ $A_{i}$, such that

$$
\frac{\left|B_{i}\right|}{\left|A_{i}\right|}>1-\varepsilon
$$


The system $\left\{A_{i}\right\}_{i=1}^{n} \varepsilon$-quasitiles $H$ if it both $\varepsilon$-covers $H$ and is $\varepsilon$-disjoint. The following result of Ornstein and Weiss [16] is crucial for our proof.

Proposition 5.1 (Quasitiling theorem). Let $F_{1} \subset F_{2} \subset \ldots$ be a Følner-sequence. Then for any $\varepsilon>0$ there exists $\delta>0$ and a finite subfamily $F_{n_{1}} \subset F_{n_{2}} \subset \cdots \subset F_{n_{k}}$ such that if $i(H)<\delta$, then $H$ can be $\varepsilon$-quasitiled by translates of the $F_{n_{i}}$ 's.

Lemma 5.1. If $A, B$ are finite subsets of a d-regular graph such that $i(A)<\varepsilon$, $B \subset A$ and $\frac{|B|}{|A|}>1-\varepsilon$, then

$$
i(B)<(d+1) \frac{\varepsilon}{1-\varepsilon} .
$$

Proof. The set $\partial B$ is covered by the union of $\partial A$ and the neighbours of the vertices in $A \backslash B$. Thus $|\partial B| \leq|\partial A|+d|A \backslash B|$. Therefore,

$$
i(B)=\frac{|\partial B|}{|B|} \leq \frac{|\partial A|+d|A \backslash B|}{|A|(1-\varepsilon)} \leq(d+1) \frac{\varepsilon}{1-\varepsilon} .
$$

The following lemma is a corollary of Lemma 5.1

Lemma 5.2. Let $\delta>0$ and $F_{1} \subset F_{2} \subset \ldots$ be a Følner-sequence. Then there exists $c_{\delta}>0$ and $n_{\delta}>0$ such that if $j \geq n_{\delta}, B \subset F_{j}$ and $\frac{|B|}{\left|F_{j}\right|}>1-c_{\delta}$, then $i(B)<\delta$.

Lemma 5.3. For any $\delta>0$, there exist finite sets $L_{1}, L_{2}, \ldots, L_{i_{\delta}} \subset \Gamma$ and a real constant $\kappa_{\delta}>0$ such that for any $1 \leq j \leq i_{\delta}$ :

- $i\left(L_{j}\right)<\delta$.

- If $i(H)<\kappa_{\delta}$, then $H$ can be tiled by translates of the $L_{i}$ 's and less than $\delta|H|$ single points.

Proof. First fix a $F \varnothing l n e r$-sequence $F_{1} \subset F_{2} \subset \ldots$ Pick an $\varepsilon>0$ such that:

- $(1-\varepsilon)^{2}>1-\delta$.

- $\epsilon<c_{\delta}$ (with $c_{\delta}$ as in the previous lemma).

Consider the subsequence $F_{n_{\delta}} \subset F_{n_{\delta}+1} \subset F_{n_{\delta}+2} \ldots$, where $n_{\delta}$ is the constant of the previous lemma. By Proposition [5.1, there exists a finite subsequence $F_{a_{1}} \subset F_{a_{2}} \subset$ $\cdots \subset F_{a_{k}}$ and a constant $\kappa_{\delta}>0$ such that if $i(H)<\kappa_{\delta}$, then $H$ can be $\varepsilon$-quasitiled by translates of the $F_{a_{i}}$ 's. By the definition of $\varepsilon$-quasitiling, for any translate $\gamma F_{a_{i}}$, we can choose a subset $R_{\gamma, i} \subset \gamma F_{a_{i}}$ in such a way that:

- The chosen subsets are disjoint.

- $\frac{\left|R_{\gamma, i}\right|}{\left|\gamma F_{a_{i}}\right|}>1-\varepsilon$.

- The union of the chosen subsets has size at least $(1-\varepsilon)^{2}|H|$.

Also, by Lemma 5.2 and the choice of $\varepsilon, i\left(R_{\gamma, i}\right)<\delta$. Therefore, if $F_{1}, F_{2}, \ldots, F_{i_{\delta}} \subset$ $\Gamma$ is just the family of all subsets of the $F_{a_{i}}$ 's having isoperimetric constant less than $\delta$, then the family and the constant $\kappa_{\delta}$ satisfy the conditions of our lemma.

Now we construct a Bratteli system by induction. Suppose that for any $1 \leq m \leq$ $n$ we have a family of tiles $\left\{F_{1}^{m}, F_{2}^{m}, \ldots, F_{i_{m}}^{m}\right\}$ and real constants $\delta_{1}>\delta_{2}>\cdots>$ $\delta_{m}>\delta_{n+1}$ such that:

- For any $1 \leq i \leq n+1, \delta_{i}<\frac{1}{2^{i}}$.

- For any $1 \leq m \leq n$ and $1 \leq j \leq i_{m}, i\left(F_{j}^{m}\right)<\delta_{m}$.

- For any $1 \leq m \leq n$, if $i(H)<\delta_{m+1}$, then $H$ can be tiled by translates of the $F_{i}^{m}$ 's and less than $\delta_{m}|H|$ single vertices. 
Now using Lemma 5.3, we choose a finite family $\left\{F_{1}^{n+1}, F_{2}^{n+1}, \ldots, F_{i_{n+1}}^{n+1}\right\}$ such that:

- $i\left(F_{j}^{n+1}\right)<\delta_{n+1}$, for any $1 \leq j \leq i_{n+1}$.

- If $i(H)<\kappa_{\delta_{n+1}}$ (see Lemma [5.3), then $H$ can be tiled by translates of the $F_{i}^{n+1}$ 's and less than $\delta_{n+1}|H|$ vertices.

Finally, set

$$
\delta_{n+2}:=\min \left\{\frac{\kappa_{\delta_{n+1}}}{2}, \frac{1}{2^{n+3}}, \frac{\delta_{n+1}}{2}\right) .
$$

By the induction above, we obtain a Bratteli system. Note that $Z_{n}$ consists of the family $\left\{F_{1}^{n}, F_{2}^{n}, \ldots, F_{i_{n}}^{n}\right\}$ and the singleton (the unit element).

Now we construct a harmonic function $m$. We fix a Følner-sequence $\left\{G_{n}\right\}_{n=1}^{\infty}$ that we will use as a tool in the rest of our paper. Let $\left\{\delta_{n}\right\}_{n=1}^{\infty}$ be the constants as above. Define $j_{n}$ such that

$$
\delta_{j_{n}+2} \leq i\left(G_{n}\right)<\delta_{j_{n}+1} .
$$

Then pick a tiling of $G_{n}$ by translates of the elements of $Z_{j_{n}}$ in such a way that the number of single vertices is less than $\left(\frac{1}{2^{j_{n}}}\right)\left|G_{n}\right|$. Then pick a tiling of the $Z_{j_{n}}$-tiles by translates of $Z_{j_{n}-1}$ in such a way that the number of single vertices in any tile $T$ is less than $\left(\frac{1}{2^{j_{n}-1}}\right)|T|$. Inductively, we obtain a tiling of $G_{n}$ by $Z_{i}$-translates for any $1 \leq i \leq j_{n}$. Note that the number of single vertices used in the $Z_{i}$-tiling of $G_{n}$ is less than

$$
\left(\sum_{k=i}^{j_{n}} \frac{1}{2^{k}}\right)\left|G_{n}\right| \leq \frac{1}{2^{i-1}}\left|G_{n}\right| .
$$

For $A \in Z_{i}$ and for $n \geq 1$ such that $j_{n} \geq i$, let us denote by $c_{n}(A)$ the number of vertices in $G_{n}$ covered by $A$-translates and let

$$
q_{n}(A)=\frac{c_{n}(A)}{\left|G_{n}\right|} .
$$

Clearly, $\sum_{A \in Z_{i}} q_{n}(A)=1$. By a diagonal argument, we can pick a subsequence $\left\{q_{n_{k}}\right\}_{k=1}^{\infty}$ such that for any tile $A, \lim _{k \rightarrow \infty} q_{n_{k}}(A)$ exists. Hence, we may suppose that for any tile $A$ the limit $m(A)=\lim _{n \rightarrow \infty} q_{n}(A)$ exists.

Lemma 5.4. The function $m$ is harmonic satisfying $\lim _{i \rightarrow \infty} m\left(E_{i}\right)=0$.

Proof. The fact that $\lim _{i \rightarrow \infty} m\left(E_{i}\right)=0$ follows from our previous observation about the number of single vertices used in the tiling of $G_{n}$. Observe that if $A \in Z_{i}$,

$$
q_{n}(A)=\sum_{B \in Z_{i+1}} \frac{S(A) K(A, B)}{S(B)} q_{n}(B) .
$$

Indeed, $\frac{q_{n}(A)\left|G_{n}\right|}{S(A)}$ is the number of $A$-tiles in $G_{n}$. Similarly, $\frac{q_{n}(B)\left|G_{n}\right|}{S(B)}$ is the number of $B$-tiles in $G_{n}$. Also, by definition, $K(A, B)$ is the number of $A$ tiles in $B$.

By taking the limit as $n \rightarrow \infty$ we get that

$$
m(A)=\sum_{B \in Z_{i+1}} \frac{S(A) K(A, B)}{S(B)} m(B) .
$$

Now let us show that our Bratteli tiling system satisfies the properties of Proposition 4.1. First we prove a simple lemma. 
Lemma 5.5. For any $n>0$ and $\delta>0$ there exists $\lambda=\lambda_{n, \delta}>0$ and $p=p_{n, \delta}>0$ with the following property. Let $l>p$ and $J \subseteq G_{l}, \frac{|J|}{\left|G_{l}\right|}>1-\lambda$. For $A \in Z_{n},|A|>1$, let $j_{A}^{l, n}$ be the number of vertices in $G_{l}$ that are covered by an A-translate (in the tiling previously defined) which is completely inside $J$. Then

$$
\left|\frac{j_{A}^{l, n}}{|J|}-m(A)\right|<\delta \text {. }
$$

Proof. The number of points covered by such $A$-translates that contain at least one point from the complement of $J$ is less than $\left|G_{l} \backslash J\right||A|$. Hence

$$
q_{l}(A)-\frac{\left|G_{l} \backslash J\right|}{\left|G_{l}\right|}|A| \leq \frac{j_{A}^{l, n}}{\left|G_{l}\right|} \leq q_{l}(A) .
$$

Since $\sup _{A \in Z_{n}}|A|<\infty$ and $q_{l}(A) \rightarrow m(A)$, the inequality of the lemma holds if $l$ is large enough and $1-\frac{|J|}{\left|G_{l}\right|}$ small enough.

Now we finish the proof of our proposition. Let $\varepsilon>0, n>0$ be the constants of our propositions and $\lambda_{n, \frac{\varepsilon}{4}}>0, p_{n, \frac{\varepsilon}{4}}>0$ be the corresponding constants in Lemma 5.5 .

Pick an $\alpha>0$ such that:

- $\alpha<\lambda_{n, \frac{\varepsilon}{4}}$.

- $1-(1-\alpha)^{2}<\frac{\varepsilon}{4 \max _{A \in Z_{n}}|A|}$.

By Proposition 5.1, we have a constant $\rho$ (note that this constant will be the $\delta_{\varepsilon, n}$ of our proposition) and a subfamily $\left\{G_{a_{1}}, G_{a_{2}}, \ldots, G_{a_{t}}\right\}$ of the Følner-sequence $\left\{G_{n}\right\}_{n=1}^{\infty}$ such that:

- $a_{1}>p_{n, \frac{\varepsilon}{4}}$

- $\left\{G_{a_{1}}, G_{a_{2}}, \ldots, G_{a_{t}}\right\} \alpha$-quasitiles any finite subset $H \subset \Gamma$ with $i(H)<\rho$.

So, let $H \subset \Gamma$ be a finite subset such that $i(H)<\rho$. Let $\bigcup_{k=1}^{s} J_{k} \subset H$ be the disjoint union of subsets, such that each $J_{k}$ covers at least $(1-\alpha)$-part of the $G_{a_{i}}{ }^{-}$ tile in our $\alpha$-quasitiling. Observe that $\sum_{k=1}^{s}\left|J_{k}\right|>(1-\alpha)^{2}|H|$. By Lemma 5.5, we can tile each $J_{k}$ by $A$-translates, $A \in Z_{n}$ such that for any $1 \leq k \leq s$,

$$
\left|c_{A}^{J_{k}}-m(A)\right| J_{k}||<\frac{\varepsilon}{4}\left|J_{k}\right|,
$$

where $c_{A}^{J_{k}}$ is the number of vertices in $J_{k}$ covered by an $A$-tile, completely inside $J_{k}$. That is,

Hence

$$
\left|\sum_{k=1}^{s} c_{A}^{J_{k}}-m(A) \sum_{k=1}^{s}\right| J_{k}|| \leq \frac{\varepsilon}{4} \sum_{k=1}^{s}\left|J_{k}\right| .
$$

$$
\left|\sum_{k=1}^{s} c_{A}^{J_{k}}-m(A)\right| H|| \leq \frac{\varepsilon}{4}|H|+\left(1-(1-\alpha)^{2}\right)|H| \leq \frac{\varepsilon}{2}|H| .
$$

Observe that for any $A \in Z_{n}$,

$$
\left|T_{A}^{H}\right|-\sum_{k=1}^{s} c_{A}^{J_{k}} \leq\left(|H|-\sum_{k=1}^{s}\left|J_{k}\right|\right)|A| \leq\left(1-(1-\alpha)^{2}\right)|H||A| \leq \frac{\varepsilon}{2}|H| .
$$

Hence, by (4), $\left|\frac{\left|T_{A}^{H}\right|}{|H|}-m(A)\right|<\epsilon$. 


\section{The Canonical Rank on amenable group algebras}

In this section we recall some results from [4. Let $\Gamma$ be a finitely generated amenable group and $K \Gamma$ be its group algebra over the field $K$. Let $\left\{F_{n}\right\}_{n=1}^{\infty}$ be a Følner-sequence. For $a \in K \Gamma$, let $V_{n}^{a} \subset K^{F_{n}} \subset K \Gamma$ be the vector space of elements $z$ supported on $F_{n}$ such that $z a=0$. Then

$$
\lim _{n \rightarrow \infty} \frac{\operatorname{dim}_{K} V_{n}^{a}}{\left|F_{n}\right|}=k_{a}
$$

exists and is independent of the choice of the Følner-sequence. We call $\operatorname{rk}(a)=1-k_{a}$ the natural rank of $a$. It is proved in [6. Proposition, page 237] that if $K=\mathbb{C}$, then $\operatorname{rk}(a)=1-\operatorname{dim}_{\Gamma} \operatorname{Ker} M_{a}$, where $\operatorname{dim}_{\Gamma}$ is the von Neumann dimension and $\operatorname{Ker} M_{a}$ is the set of elements $w \in l^{2}(\Gamma)$ for which $w a=0$. Note that the rank can be computed slightly differently as well. Let $S$ be a symmetric generating system of $\Gamma$ and $\operatorname{Cay}(\Gamma, S)$ be the Cayley-graph of $\Gamma$. We consider the shortest path metric $d_{C a y(\Gamma, S)}$ on $\Gamma$. Choose $r$ such that $\operatorname{supp}(a) \subset B_{r}(1)$, where $B_{r}(1)$ is the $r$-ball around the unit element in the Cayley-graph and

$$
\operatorname{supp}(a)=\left\{\gamma \in \Gamma \mid a_{\gamma} \neq 0\right\}
$$

if $a=\sum a_{\gamma} \gamma$. For a finite set $F \subset \Gamma$, let $\partial_{r} F$ be the set of elements $x \in F$ such that

$$
d_{C a y(\Gamma, S)}\left(x, F^{c}\right) \leq r .
$$

Note that $\partial F=\partial_{1}(F)$. Clearly, if $b \in K \Gamma$ and $\operatorname{supp}(b) \in F \backslash \partial_{r} F$, then $\operatorname{supp}(b a) \subset$ $F$. Then for any $s>r$, we have the following lemma.

\section{Lemma 6.1.}

$$
r k(a)=\lim _{n \rightarrow \infty} \frac{\operatorname{dim}_{K} W_{F_{n}} a}{\left|F_{n}\right|}
$$

where $W_{F_{n}}$ is the set of elements in $K \Gamma$ supported on $F_{n} \backslash \partial_{s} F_{n}$.

Proof. By our definition, $\operatorname{rk}(a)=\lim _{n \rightarrow \infty} \frac{\operatorname{dim}_{K} K^{F_{n}} a}{\left|F_{n}\right|}$. Observe that

$$
\frac{\operatorname{dim}_{K} K^{F_{n}} a-\operatorname{dim}_{K} W_{F_{n}} a}{\left|F_{n}\right|} \leq \frac{\operatorname{dim}_{K} K^{F_{n}}-\operatorname{dim}_{K} W_{F_{n}}}{\left|F_{n}\right|}=\frac{\left|\partial_{s} F_{n}\right|}{\left|F_{n}\right|} .
$$

Since $\lim _{n \rightarrow \infty} \frac{\left|\partial_{s} F_{n}\right|}{\left|F_{n}\right|}=0$, the lemma follows.

\section{SOFIC REPRESENTATIONS}

7.1. Sofic approximation and sofic representations. In this section we recall the notion of sofic representations from [7. Let $\Gamma$ be a finitely generated group with a symmetric generating set $S$. Let $\left\{P_{n}\right\}_{n=1}^{\infty}$ be a sequence of finite graphs such that the directed edges are labeled by the elements of $S$ in such a way that if $(x, y)$ is labeled by $s$, then $(y, x)$ is labeled by $s^{-1}$. We say that $\left\{P_{n}\right\}_{n=1}^{\infty}$ is a sofic approximation of $\Gamma$ if for any natural number $r>0$ there exists $n_{r}>0$ such that:

- If $n \geq n_{r}$, then for the set $V_{n}^{r}$ of vertices $x \in P_{n}$ for which the ball $B_{r}(x)$ in $P_{n}$ is isomorphic to the ball $B_{r}(1) \in \operatorname{Cay}(\Gamma, S)$ as labeled graphs

$$
\frac{\left|V_{n}^{r}\right|}{\left|P_{n}\right|}>1-\frac{1}{r}
$$


A group is called sofic if it possesses a sofic approximation. At the moment, no non-sofic group is known. If $\operatorname{Cay}(\Gamma, S)$ is the Cayley-graph of an amenable group and $\left\{F_{n}\right\}_{n=1}^{\infty}$ is a Følner-sequence, then the induced graphs $P_{n}$ of the sets $F_{n}$ form a sofic approximation of $\Gamma$. If $\Gamma$ is residually finite (amenable or not) with normal chain $\left\{N_{k}\right\}_{k=1}^{\infty}, \bigcap_{k=1}^{\infty} N_{k}=\{1\}$, then the graph sequence $\operatorname{Cay}\left(\Gamma / N_{k}, S\right)$ forms a sofic approximation of $\Gamma$. If $\left\{P_{n}\right\}_{n=1}^{\infty}$ is an arbitrary sofic approximation of a group $\Gamma$, then one can construct an embedding of $K \Gamma$ ( $K$ is an arbitrary field) to the ultraproduct of matrix algebras the following way.

Let $\left\{\operatorname{Mat}_{P_{n} \times P_{n}}(K)\right\}_{n=1}^{\infty}$ be a sequence of matrix algebras. Let $a \in K \Gamma, a=$ $\sum a_{\gamma} \gamma$ be an element of the group algebra and choose $r$ such that if $a_{\gamma} \neq 0$, then $\gamma \in B_{r}(1) \subset \operatorname{Cay}(\Gamma, S)$. Let $\left\{e_{x}\right\}_{x \in P_{n}}$ be the natural basis of $K^{P_{n}}$. If $x \in V_{n}^{r}$, then let

$$
\psi_{n}(a)\left(e_{x}\right)=\sum_{\gamma \in B_{r}(1)} a_{\gamma} e_{x \gamma} .
$$

Note that by our condition on the support of $a$, and because the $r$-ball around $x$ is isomorphic as labeled graphs to the corresponding ball in the Cayley graph of $\Gamma, x \gamma=y$ is meaningful. If $x \notin V_{n}^{r}$, let $\psi_{n}(a)\left(e_{x}\right)=0$. This way one defines an injective homomorphism $\psi_{\mu}: K \Gamma \rightarrow \mathcal{M}_{\mu}^{\text {alg }}$, where $\mu=\left\{\left|P_{1}\right|,\left|P_{2}\right|, \ldots\right\}$ ([7], Theorem 1). If $K=\mathbb{C}$, the homomorphism above is a $*$-homomorphism. The map $\psi_{\mu}$ is called the sofic representation associated to the sequence $\left\{P_{n}\right\}_{n=1}^{\infty}$.

7.2. Sofic approximation of amenable groups. Let $\left\{P_{n}\right\}_{n=1}^{\infty}$ be a sofic approximation of the amenable group $\Gamma$ (with symmetric generating set $S$ ). For $L>0$, let $V_{n}^{L}$ be the set of vertices $x$ in $P_{n}$ such that

$$
B_{L}(x) \cong B_{L}(1) \subset \operatorname{Cay}(\Gamma, S)
$$

as $S$-labeled graphs. If $F \subset B_{L}(1)$, then for $x \in V_{n}^{L}$ we call $\pi(F)$ an $F$-translate, where $\pi: B_{L}(1) \rightarrow B_{L}(x)$ is the $S$-labeled graph isomorphism mapping 1 to $x$. In [8, Theorem 2] we proved the following generalization of the Ornstein-Weiss quasitiling theorem.

Proposition 7.1 (Generalized quasitiling theorem). Let $F_{1} \subset F_{2} \ldots$ be a Følnersequence. Then for any $\varepsilon>0$ there exists $L>0, \delta>0$ and a finite subcollection $F=\left\{F_{n_{1}}, F_{n_{2}}, \ldots, F_{n_{t}}\right\}$ such that if

$$
\frac{\left|V_{n}^{L}\right|}{\left|P_{n}\right|}>1-\delta
$$

then $P_{n}$ can be $\varepsilon$-quasitiled by $F$-translates.

\section{Embedding $K \Gamma$ to the COMPletion of An ultramatricial algebra}

Let $\left(\left\{Z_{i}\right\}_{i=1}^{\infty}, m\right)$ be a Bratteli tiling system as in Proposition 4.1. Let $E_{B}, \phi_{i}$ be as in Section 4 , and $\mathcal{A}_{\phi}$ be the ultramatricial algebra corresponding to the system $\left(\left\{Z_{i}\right\}_{i=1}^{\infty}, m\right)$. Remember that any $A \in Z_{i}$ is in fact some finite subset of $\Gamma$.

Now we define the maps $\pi_{i}^{A}: K \Gamma \rightarrow \operatorname{Mat}_{A \times A}(K)$ the following way. Recall from Section 4 that we regard the elements of $\operatorname{Mat}_{A \times A}(K)$ as kernel maps. Let $a=\sum a_{\gamma} \gamma \in K \Gamma$, and choose $r$ minimal with $\operatorname{supp}(a) \subset B_{r}(1)$. If $x \in A \backslash \partial_{r}(A)$, then let

$$
\pi_{i}^{A}(a)\left(e_{x}\right)=\sum a_{\gamma} e_{x \gamma}
$$


Note that by the condition on the support $x \gamma$ is well-defined. If $x \in \partial_{r}(A)$, then let $\pi_{i}^{A}(a)\left(e_{x}\right)=0$. Finally we define the maps $\pi_{i}:=\bigoplus_{A \in Z_{i}} \pi_{i}^{A}: K \Gamma \rightarrow$ $\bigoplus_{A \in Z_{i}} \operatorname{Mat}_{A \times A}(K)$. Thus if $|A|=1$, then $\pi_{i}^{A}(a)=0$.

Lemma 8.1. For any $a \in K \Gamma,\left\{\left[\pi_{i}(a)\right]\right\}_{i=1}^{\infty}$ is a Cauchy-sequence in $\mathcal{A}_{\phi}$, where $\left[\pi_{i}(a)\right]$ denotes the image of $\pi_{i}(a)$ under the natural embedding $\bigoplus_{A \in Z_{i}} \operatorname{Mat}_{A \times A}(K)$ $\rightarrow \mathcal{A}_{\phi}$.

Proof. First of all note that

$$
\operatorname{rk}_{\phi}\left(\phi_{i} \circ \pi_{i}(a)-\pi_{i+1}(a)\right)=\sum_{B \in Z_{i+1}} m_{i+1}(B) \frac{\operatorname{rank}\left(E_{B} \circ \pi_{i}(a)-\pi_{i+1}^{B}(a)\right)}{|B|} .
$$

Observe that

$$
\operatorname{rank}\left(E_{B} \circ \pi_{i}(a)-\pi_{i+1}^{B}(a)\right)=|B|-\operatorname{dim}_{K} \operatorname{ker}\left(E_{B} \circ \pi_{i}(a)-\pi_{i+1}^{B}(a)\right) .
$$

On the other hand,

$$
\operatorname{dim}_{K} \operatorname{ker}\left(E_{B} \circ \pi_{i}(a)-\pi_{i+1}^{B}(a)\right) \geq T_{B},
$$

where $T_{B}$ is the number of vertices $x$ in $B$ for which

$$
E_{B} \circ \pi_{i}(a)\left(e_{x}\right)=\pi_{i+1}^{B}(a)\left(e_{x}\right) .
$$

Clearly,

$$
T_{B} \geq|B|-\left|\partial_{r} B\right|-\sum_{A \in Z_{i}} K(A, B)\left|\partial_{r}(A)\right| .
$$

Recall that if $|B|>1$, then $|\partial B| \leq|B| 2^{-(i-1)}$. Indeed, $B$ is tiled with tiles of isoperimetric constants less than $\frac{1}{2^{i}}$ and at most $\frac{1}{2^{i+1}}|B|$ singletons. Hence

$$
\left|\partial_{r} B\right| \leq|B| 2^{-(i-1)}(d+1)^{r+1},
$$

where $d$ is the degree of the vertices in the Cayley graph. Also,

$$
\sum_{A \in Z_{i}} K(A, B)\left|\partial_{r}(A)\right| \leq K\left(E_{i}, B\right)+\sum_{A \in Z_{i},|A|>1} K(A, B)|A| 2^{-(i-2)}(d+1)^{r+1} .
$$

Therefore,

$$
T_{B} \geq|B|-|B| 2^{-(i-1)}(d+1)^{r+1}-|B| 2^{-(i+1)}-|B| 2^{-(i-2)}(d+1)^{r+1} .
$$

Hence,

$$
\begin{aligned}
& \operatorname{rk}_{\phi}\left(\phi_{i} \circ \pi_{i}(a)-\pi_{i+1}(a)\right) \leq 2^{-(i+1)} \\
& \quad+\sum_{B \in Z_{i+1},|B|>1} m_{i+1}(B)\left(2^{-(i-1)}(d+1)^{r+1}+2^{-(i+1)}+2^{-(i-2)}(d+1)^{r+1}\right) .
\end{aligned}
$$

Thus the lemma follows.

Lemma 8.2. Let $a \in K \Gamma, b \in K \Gamma$, then:

(1) $\lim _{i \rightarrow \infty} r k_{\phi}\left(\pi_{i}(a) \pi_{i}(b)-\pi_{i}(a b)\right)=0$.

(2) $\lim _{i \rightarrow \infty} r k_{\phi}\left(\pi_{i}(a)+\pi_{i}(b)-\pi_{i}(a+b)\right)=0$.

(3) If $K=\mathbb{C}$, then $\lim _{i \rightarrow \infty} r k_{\phi}\left(\pi_{i}\left(a^{*}\right)-\pi_{i}(a)^{*}\right)=0$. 
Proof. We prove only the first part; the other two statements can be seen in exactly the same way. If $x \in A \backslash \partial_{r+s}(A)$, where $\operatorname{supp}(a) \subset B_{r}(1)$ and $\operatorname{supp}(b) \subset B_{s}(1)$, then

$$
\left(\pi_{i}^{A}(a) \pi^{A}(b)-\pi_{i}^{A}(a b)\right)\left(e_{x}\right)=0
$$

Therefore

$$
\operatorname{rk}_{\phi}\left(\pi_{i}(a) \pi_{i}(b)-\pi_{i}(a b)\right) \leq \sum_{A \in Z_{i}} m(A) \frac{\left|\partial_{r+s}(A)\right|}{|A|} \leq \frac{1}{2^{i}}+\max _{A \in Z_{i}|A|>1} \frac{\left|\partial_{r+s}(A)\right|}{|A|} .
$$

Since $\lim _{i \rightarrow \infty} \max _{A \in Z_{i}|A|>1} \frac{\left|\partial_{r+s}(A)\right|}{|A|}=0$, the lemma follows.

Let $\pi(a) \in \overline{\mathcal{A}}_{\phi}$ be the limit of elements $\pi_{i}(a)$ in the rank completion of the ultramatricial algebra $\mathcal{A}_{\phi}$. By the previous lemma $\pi$ is a homomorphism and if $K=\mathbb{C}$, then $\pi$ is even a $\star$-homomorphism. Finally, we prove that

$$
\operatorname{rk}_{\phi}(\pi(a))=\operatorname{rk}_{\Gamma}(a) .
$$

By definition,

$$
\operatorname{rk}_{\phi}\left(\pi_{i}(a)\right)=\sum_{A \in Z_{i}} m(A) \frac{\operatorname{rank}\left(\pi_{i}^{A}(a)\right)}{|A|}
$$

If $|A|=1$, then $m(A) \leq 2^{-i}$. By Lemma 6.1, $\lim _{i \rightarrow \infty} \frac{\operatorname{rank}\left(\pi_{i}^{A_{i}}(a)\right)}{\left|A_{i}\right|}=\operatorname{rk}_{\Gamma}(a)$, where $A_{i}$ is an arbitrary element of $Z_{i}$ such that $\left|A_{i}\right|>1$. Hence by (15), $\operatorname{rk}_{\phi}(\pi(a))=$ $\mathrm{rk}_{\Gamma}(a)$. This finishes the proof of Theorem 2 .

Remark. Let $\Gamma$ be a residually finite amenable group and $K$ be a field such that $K \Gamma$ has no zero-divisor. Let $\Gamma=N_{0} \supset N_{1} \supset N_{2} \ldots, \bigcap_{k=1}^{\infty} N_{k}=\{1\}$ be finite index normal subgroups and $\pi_{k}: \mathbb{C} \Gamma \rightarrow \mathbb{C}\left(\Gamma \backslash N_{k}\right)$ be the natural projections as in Subsection 1.3. Then the following version of (2) holds by [14, Theorem $0.2(\mathrm{i})$ ] (the authors use a slightly different language). For any $\Delta \in \operatorname{Mat}_{d \times d}(K \Gamma)$,

$$
\lim _{k \rightarrow \infty} \frac{\operatorname{dim}_{K} \operatorname{Ker}\left(\pi_{k}(\Delta)\right)}{\left|\Gamma: N_{k}\right|}=d-\operatorname{rk}_{S^{-1} K \Gamma}(i(\Delta))
$$

where the skewfield $S^{-1} K \Gamma$ is the Ore localization of $K \Gamma$ and $i$ is the natural embedding. One can actually prove, using similar methods, that for arbitrary finitely generated amenable residually finite groups

$$
\lim _{k \rightarrow \infty} \frac{\operatorname{dim}_{K} \operatorname{Ker}\left(\pi_{k}(\Delta)\right)}{\left|\Gamma: N_{k}\right|}=d-\operatorname{rk}_{\mathcal{A}_{\phi}}(\pi(\Delta))
$$

where $\operatorname{rk}_{\mathcal{A}_{\phi}}$ is the matrix rank in $\operatorname{Mat}_{d \times d}\left(\mathcal{A}_{\phi}\right)$.

\section{The proof of Theorem 1}

In this section we prove the three main identities of Section 2 finishing the proof of Theorem 1 . 
9.1. The first identity. We will prove that $f_{5} \circ f_{4}=f_{1}$, where:

- $f_{1}$ is the map $\psi_{\mu}$ defined in Subsection 7.1 (associated to the sofic approximation below).

- $f_{4}=\pi$, where $\pi: \mathbb{C} \Gamma \rightarrow \overline{\mathcal{A}_{\phi}}$ is the map defined in Section 8 .

- The map $f_{5}$ will be defined below.

Let $\left\{P_{n}\right\}_{n=1}^{\infty}$ be the sofic approximation of our group $\Gamma$. Our first goal is to construct a map

$$
\tau: \mathcal{A}_{\phi} \rightarrow \prod_{n=1}^{\infty} \operatorname{Mat}_{P_{n} \times P_{n}}(\mathbb{C}),
$$

where $\mathcal{A}_{\phi}$ is the ultramatricial algebra of Section 4. We fix a Bratteli tiling system $\left(\left\{Z_{n}\right\}_{n=1}^{\infty}, m\right)$ for the whole section.

Lemma 9.1. We have a sequence $\{j(n)\}_{n=1}^{\infty}, \lim _{n \rightarrow \infty} j(n)=\infty$ with the following properties:

- We have an iterated tiling of $P_{n}$ starting with a $Z_{j(n)}$-tiling.

- For any $k>0$ and $A \in Z_{k}$,

$$
\lim _{n \rightarrow \infty} \frac{\left|T_{A}^{P_{n}}\right|}{\left|P_{n}\right|}=m(A),
$$

where $T_{A}^{P_{n}}$ is the number of points in $P_{n}$ covered by A-tiles in the $Z_{k}$-tiling of $P_{n}$.

Proof. Let $G_{1} \subset G_{2} \subset \ldots$ be the Følner-sequence in the proof of Proposition 4.1 . Recall that for each $n \geq 1$ there exist inductive tilings of the $G_{n}$ 's such that

$$
\lim _{n \rightarrow \infty} \frac{\left|T_{A}^{G_{n}}\right|}{\left|G_{n}\right|}=m(A) .
$$

Claim 9.1. We have a sequence $\{\varepsilon(n)\}_{n=1}^{\infty}$ tending to zero and for each $n \geq 1$ a subfamily $G^{n}=\left\{G_{t_{1}^{n}} \subset G_{t_{2}^{n}} \subset \cdots \subset G_{t_{m_{n}}^{n}}\right\}$ such that:

- $P_{k}$ is $\varepsilon(n)$-quasitiled by $G^{n}$-tiles, if $k \geq n$.

- $\lim _{n \rightarrow \infty} t_{1}^{n}=\infty$.

Proof. We define inductively the sequences $\{\epsilon(n)\}_{n=1}^{\infty} \subset \mathbb{R}^{+},\left\{c_{n}\right\}_{n=1}^{\infty} \subset \mathbb{N}$ and $\left\{t_{1}^{n}\right\}_{n=1}^{\infty} \in \mathbb{N}$ such that $\epsilon(n) \rightarrow 0, t_{1}^{n} \rightarrow \infty$. First of all, let $\epsilon(1):=1, t_{1}^{1}:=1$, $c_{1}:=2$. Moreover, given the Følner-sequence $G_{n} \subset G_{n+1} \subset \ldots$ and $\epsilon=1 / n$, let $L_{n}>0, \delta_{n}>0$ be the corresponding constants, and $F^{n}$ be the subcollection of the Følner-sequence as in Proposition 7.1. Also, let $N_{n}$ be the natural number in the definition of the sofic approximation such that $P_{k}$ can be $1 / n$-quasitiled by $F^{n}$-translates for any $k \geq N_{n}$, as in Proposition 7.1. Since everything can be 1quasitiled by any tiling system, we can clearly assume that $N_{1}=1$. Moreover, we can choose $G^{1}$ arbitrarily.

Now we make the inductive definition. Suppose that for $i \leq n-1, \varepsilon(i), c_{i}, G^{i}$ are already defined and $P(k)$ can be $\varepsilon(i)$-quasitiled by $G^{i}$-translates if $k \geq 1$. If $n<N_{c_{n-1}}$, we set $c_{n}:=c_{n-1}, \epsilon(n):=\epsilon(n-1)$ and $G^{n}:=G^{n-1}$. However, if $n \geq N_{c_{n-1}}$, then we set $c_{n}:=c_{n-1}+1, \epsilon(n):=1 / c_{n-1}, G^{n}:=F^{c_{n-1}}$. It follows from the choices that for any $k \geq n, P(k)$ can be $\epsilon(n)$-quasitiled by $G^{n}$-tiles and $t_{1}^{n} \geq c_{n-1}$. Hence we get the desired sequences. 
In the next step, we construct the numbers $j(n)$ and the iterated tilings of the sets $P_{n}$, starting with a $Z_{j(n)}$-tiling. Recall that, for each $n$, we have in Section 5 defined $j_{n}$ and an iterated $Z_{j_{n}}$-tiling of $G_{n}$ with the properties we are interested in. We wish to make use of these tilings for the $G^{n}$-quasitiles of the quasitiling of $P_{n}$ we have found. Choose $j(n)$ maximal such that $j(n) \leq j_{t_{1}^{n}}, \ldots, j \leq j_{t_{m_{n}}^{n}}$ and $|A| \leq 1 / \sqrt{\epsilon(n)}$ for each $A \in Z_{j(n)}$. Since $\epsilon_{n} \rightarrow 0$ and all the $t_{k}^{n} \geq t_{1}^{n} \rightarrow \infty$, it immediately follows that $j(n) \rightarrow \infty$.

By the definition of the quasitiling, we have disjoint subsets $\left\{J_{\alpha}\right\}_{\alpha \in I_{n}}$ such that each $J_{\alpha}$ is inside a $G^{n}$-translate $L_{\alpha}$, covering at least the $(1-\varepsilon(n))$ part of $L_{\alpha}$. For any $J_{\alpha} \subset L_{\alpha}$, we consider an iterated tiling. First, we take the $Z_{j(n)}$-tiling of $L_{\alpha}$,

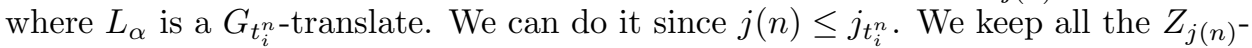
tiles that are completely covered by $J_{\alpha}$ and cover all other points in $J_{\alpha}$ by singletons. Then we start the iterative tiling procedure. That is, for any $1 \leq i \leq j(n)$, the $Z_{i}$-tiling of $J_{\alpha}$ consists of the $Z_{i}$-tiles of $L_{\alpha}$ such that the corresponding $Z_{j(n)}$-tile is completely in $J_{\alpha}$ and some singletons.

Lemma 9.2. For any index sequence $\left\{\alpha_{n} \in I_{n}\right\}_{n=1}^{\infty}$, for any $i \geq 1$ and $A \in Z_{i}$,

$$
\lim _{n \rightarrow \infty} \frac{\left|T_{A}^{J_{\alpha_{n}}}\right|}{\left|J_{\alpha_{n}}\right|}=m(A),
$$

where $T_{A}^{J_{\alpha_{n}}}$ is the number of vertices in $J_{\alpha_{n}}$ covered by an A-tile in the $Z_{i}$-tiling of $J_{\alpha_{n}}$.

Proof. We use the same idea as in the proof of Lemma 5.5. The number of vertices in $L_{\alpha_{n}}$ that are in a $Z_{j(n)}$-tile not completely in $J_{\alpha_{n}}$ is less than $\left|L_{\alpha_{n}} \backslash J_{\alpha_{n}}\right| M$, where $M$ is the maximal size of a $Z_{j(n)}$-tile. Hence the lemma follows from our condition on the maximal size of a $Z_{j(n)}$-tile.

One can immediately see that Lemma 9.1 follows from Lemma 9.2 .

Recall from Section 4 the construction of an injective homomorphism

$$
E_{P}: \bigoplus_{A \in Z_{k}} \operatorname{Mat}_{A \times A}(\mathbb{C}) \rightarrow \operatorname{Mat}_{P \times P}
$$

associated to a $Z_{k}$-tiling of a graph $P$. Now if $i\left(P_{n}\right) \geq k$, then let

$$
\tau_{k, n}: \bigoplus_{A \in Z_{k}} \operatorname{Mat}_{A \times A}(\mathbb{C}) \rightarrow \operatorname{Mat}_{P_{n} \times P_{n}}
$$

is the homomorphism associated to the $Z_{k}$-tiling of $P_{n}$ given in Lemma 9.1. By the iterative tiling construction, if $j\left(P_{n}\right) \geq k+1$, then

$$
\tau_{k, n}=\tau_{k+1, n} \circ \phi_{k},
$$

where $\phi_{k}: \bigoplus_{A \in Z_{k}} \operatorname{Mat}_{A \times A}(\mathbb{C}) \rightarrow \bigoplus_{B \in Z_{k+1}} \operatorname{Mat}_{B \times B}(\mathbb{C})$ is the injective homomorphism defined in Section 4 . Let us define $\tau_{k, n}$ to be the zero homomorphism if $k>j\left(P_{n}\right)$. Hence, we defined a homomorphism

$$
\tau^{k}=\left(\tau_{k, 1}, \tau_{k, 2} \ldots\right): \bigoplus_{A \in Z_{k}} \operatorname{Mat}_{A \times A}(\mathbb{C}) \rightarrow \prod_{n=1}^{\infty} \operatorname{Mat}_{P_{n} \times P_{n}}
$$


Lemma 9.3. For any $\bigoplus_{A \in Z_{k}} a_{A} \in \bigoplus_{A \in Z_{k}} \operatorname{Mat}_{A \times A}(\mathbb{C})$,

$$
\lim _{n \rightarrow \infty} \frac{\operatorname{rank}\left(\tau_{k, n}\left(\bigoplus_{A \in Z_{k}} a_{A}\right)\right)}{\left|P_{n}\right|}=r k_{\phi}\left(\bigoplus_{A \in Z_{k}} a_{A}\right) .
$$

Proof.

$$
\begin{gathered}
\lim _{n \rightarrow \infty} \frac{\operatorname{rank}\left(\tau_{k, n}\left(\bigoplus_{A \in Z_{k}} a_{A}\right)\right)}{\left|G_{n}\right|}=\lim _{n \rightarrow \infty} \sum_{A \in Z_{k}} \frac{\frac{\left|T_{A}^{P_{n}}\right|}{|A|} \operatorname{rank}\left(a_{A}\right)}{\left|P_{n}\right|} \\
=\sum_{A \in Z_{k}} m(A) \frac{\operatorname{rank}\left(a_{A}\right)}{|A|}=\operatorname{rk}_{\phi}\left(\bigoplus_{A \in Z_{k}} a_{A}\right) .
\end{gathered}
$$

Let $\mathcal{Q} \subset \prod_{n=1}^{\infty} \operatorname{Mat}_{P_{n} \times P_{n}}$ be the ideal of elements that are eventually zero. Clearly, $\mathcal{Q} \subset \mathcal{I}$ and $\mathcal{Q} \subset \mathcal{J}$, where $\mathcal{I}$ and $\mathcal{J}$ are the ideals introduced in Subsection 1.2. By (6), $\operatorname{Im}\left(\tau^{k}-\tau^{k+1} \circ \phi_{k}\right) \in \mathcal{Q}$. Hence, the $\tau^{k}$ 's extend to a map $\tau: \mathcal{A}_{\phi} \rightarrow$ $\prod_{n=1}^{\infty} \operatorname{Mat}_{P_{n} \times P_{n}} / \mathcal{Q}$. By Lemma 9.3, the map $\tau_{a l g}=\pi \circ \tau: \mathcal{A}_{\phi} \rightarrow \mathcal{M}_{\mu}^{\text {alg }}$ is rankpreserving, where

$$
\pi: \prod_{n=1}^{\infty} \operatorname{Mat}_{P_{n} \times P_{n}} / \mathcal{Q} \rightarrow \mathcal{M}_{\mu}^{\text {alg }}
$$

is the natural quotient map. Therefore $\tau_{a l g}$ can be extended to the completion $\overline{\mathcal{A}_{\phi}}$. That will be our map $f_{5}: \overline{\mathcal{A}_{\phi}} \rightarrow \mathcal{M}_{\mu}^{a l g}$.

Now let us prove $f_{1}=f_{5} \circ f_{4}$. Let $a \in \mathbb{C} \Gamma, \operatorname{supp}(a) \subset B_{r}(1) \subset \operatorname{Cay}(\Gamma, S)$. Then $f_{1}(a)$ can be represented in $\prod_{n=1}^{\infty} \operatorname{Mat}_{P_{n} \times P_{n}}(\mathbb{C})$ by the element $\prod_{n=1}^{\infty} \psi_{n}(a)$, where $\psi_{n}$ is defined in Subsection 7.1

Recall from Section 8 that $f_{4}(a)=\lim _{k \rightarrow \infty} \pi_{k}(a)$. Since $f_{5}$ is rank preserving, $f_{5} \circ f_{4}(a)$ equals to $\lim _{k \rightarrow \infty} f_{5} \circ \pi_{k}(a)$. Observe that $f_{5} \circ \pi_{k}(a)$ is represented in $\prod_{n=1}^{\infty} \operatorname{Mat}_{P_{n} \times P_{n}}(\mathbb{C})$ by

$$
\tau^{k} \circ \pi_{k}(a)=\prod_{n=1}^{\infty} \psi_{n}^{k}(a)
$$

where

- $a=\sum a_{\gamma} \gamma$ and $r>0$ is the smallest number such that $\operatorname{supp}(a) \subset B_{r}(1)$.

- $\psi_{n}^{k}(a)=0$, if $j(n)<k$.

- $\psi_{n}^{k}(a)\left(e_{x}\right)=\sum_{y \in B_{r}(1)} a_{\gamma} e_{x \gamma}$, if $j(n) \geq k$ and $x \in S \backslash \partial_{r} S$ for some $Z_{k^{-}}$ translate $S$ in the $Z_{k}$-tiling of $P_{n}$.

Observe that in $\mathcal{M}_{\mu}^{a l g}$,

$$
\operatorname{rank}\left[\prod_{n=1}^{\infty}\left(\psi_{n}(a)-\psi_{n}^{k}(a)\right)\right] \leq 1-\lim _{\omega} \frac{\left|z_{n}^{k}(a)\right|}{\left|P_{n}\right|},
$$

where $z_{n}^{k}(a)$ is the set of elements $x \in G_{n}$ for which $\psi_{n}^{k}(a)\left(e_{x}\right)=\psi_{n}(a)\left(e_{x}\right)$. Therefore $f_{1}=f_{5} \circ f_{4}$ immediately follows from the lemma below.

Lemma 9.4. $\lim _{k \rightarrow \infty} \liminf _{n \rightarrow \infty} \frac{\left|P_{n} \backslash z_{n}^{k}(a)\right|}{\left|P_{n}\right|}=0$. 
Proof. If $x \in P_{n} \backslash z_{n}^{k}(a)$, then either $x \in \partial_{r} S$, for some $Z_{k}$-translate $S,|S|>1$ or $x$ is a singleton in the $Z_{k}$-tiling of $P_{n}$. Fix a real constant $\varepsilon>0$. By the definition of the Bratteli tiling systems, if $k$ is large enough, then $m_{k}\left(E_{k}\right)<\frac{\varepsilon}{2}$. Hence by Lemma 9.1, for $n$ large enough (depending on $k$ ), the number of singletons in the $Z_{k}$-tiling of $P_{n}$ is less than $\frac{\varepsilon}{2}\left|P_{n}\right|$. On the other hand, by the definition of the Bratteli tiling systems, for $k$ large enough, $\frac{\left|\partial_{r}(A)\right|}{|A|}<\frac{\varepsilon}{2}$, if $A \in Z_{k}$ and $|A|>1$. Hence the lemma follows.

9.2. The second identity. We need to prove that $f_{6} \circ f_{4}=f_{3}$, where $f_{4}$ is defined in the previous subsection. The maps $f_{3}$ and $f_{6}$ will be defined below.

Let $\mathrm{rk}_{1}$, resp. $\mathrm{rk}_{2}$, denote the ranks on $\mathcal{M}_{\mu}$, resp. $\mathcal{M}_{\mu}^{\text {alg }}$. Let

$$
t \in \prod_{n=1}^{\infty} \operatorname{Mat}_{P_{n} \times P_{n}}(\mathbb{C})=\left(t_{1}, t_{2}, \ldots\right),
$$

where $\sup _{n \geq 1}\left\|t_{n}\right\|<\infty$. Note that $t$ represents an element $[t]_{\mathcal{M}_{\mu}}$ in $\mathcal{M}_{\mu}$ and an element $[t]_{\mathcal{M}_{\mu}^{a l g}}$ in $\mathcal{M}_{\mu}^{\text {alg }}$. It is important to note that $\operatorname{rk}_{1}\left([t]_{\mathcal{M}_{\mu}}\right)$ is not necessarily equal to $\operatorname{rk}_{2}\left([t]_{\mathcal{M}_{\mu}^{a l g}}\right)$. Indeed, let $t_{n}=\frac{1}{n} I d$. Then $[t]_{\mathcal{M}_{\mu}}=0$, hence $\operatorname{rk}_{1}\left([t]_{\mathcal{M}_{\mu}}\right)=0$. Nevertheless, $\operatorname{rk}_{2}\left([t]_{\mathcal{M}_{\mu}^{a l g}}\right)=1$. However, we have the following lemma.

Lemma 9.5. Let $t=\left(t_{1}, t_{2}, \ldots\right) \in \prod_{n=1}^{\infty} \operatorname{Mat}_{P_{n} \times P_{n}}(\mathbb{C})$, where for any $n \geq 1$, $t_{n}$ is self-adjoint and all the $t_{n}$ 's together have finitely many distinct eigenvalues $\lambda_{0}=0, \lambda_{1}, \lambda_{2}, \ldots, \lambda_{k}$. Then

$$
r k_{1}\left([t]_{\mathcal{M}_{\mu}}\right)=r k_{2}\left([t]_{\mathcal{M}_{\mu}^{a l g}}\right) .
$$

Proof. Let $t_{n, i}$ be the multiplicity of $\lambda_{i}$ in $t_{n}$. Then

$$
\operatorname{rk}_{2}\left([t]_{\mathcal{M}_{\mu}^{a l g}}\right)=\lim _{\omega}\left(1-\frac{t_{n, 0}}{\left|P_{n}\right|}\right) .
$$

The spectral decomposition of $[t]_{\mathcal{M}_{\mu}}$ is $\sum_{i=1}^{k} \lambda_{i} P_{i}$, where

$$
\operatorname{tr}_{\mathcal{M}_{\mu}}\left(P_{i}\right)=\lim _{\omega}\left(1-\frac{t_{n, i}}{\left|P_{n}\right|}\right) \text {. }
$$

By (1),

In general, we have the following inequality.

$$
\operatorname{rk}_{1}\left([t]_{\mathcal{M}_{\mu}}\right)=\lim _{\omega}\left(1-\frac{t_{n, 0}}{\left|P_{n}\right|}\right) .
$$

Proposition 9.1. Let $t=\left(t_{1}, t_{2}, \ldots\right) \in \prod_{n=1}^{\infty} \operatorname{Mat}_{P_{n} \times P_{n}}(\mathbb{C})$ such that $\sup _{n \geq 1}\left\|t_{n}\right\|$ $\leq K$. Then

$$
r k_{1}\left([t]_{\mathcal{M}_{\mu}}\right) \leq r k_{2}\left([t]_{\mathcal{M}_{\mu}^{a l g}}\right) .
$$

Proof. Denote by $R_{n}$ the orthogonal projection onto the kernel of $t_{n}^{*} t_{n}$. Consider the projection $R=\left(R_{1}, R_{2}, \ldots\right) \in \prod_{n=1}^{\infty} \operatorname{Mat}_{P_{n} \times P_{n}}(\mathbb{C})$. By definition,

$$
\operatorname{rk}_{2}\left([t]_{\mathcal{M}_{\mu}^{a l g}}\right)=\operatorname{rk}_{2}\left(\left[t^{*} t\right]_{\mathcal{M}_{\mu}^{a l g}}\right)=1-\operatorname{rk}_{2}\left([R]_{\mathcal{M}_{\mu}^{a l g}}\right) .
$$

By Lemma 9.5, $\operatorname{rk}_{2}\left([R]_{\mathcal{M}_{\mu}^{a l g}}\right)=\operatorname{rk}_{1}\left([R]_{\mathcal{M}_{\mu}}\right)$. Hence $\operatorname{rk}_{2}\left([t]_{\mathcal{M}_{\mu}^{\text {alg }}}\right)=1-\operatorname{rk}_{1}\left([R]_{\mathcal{M}_{\mu}}\right)$.

Lemma 9.6. If $E_{\lambda}, \lambda>0$ is an orthogonal projection in the spectral decomposition of $\left[t^{*} t\right]_{\mathcal{M}_{\mu}}$, then $[R]_{\mathcal{M}_{\mu}} \leq E_{\lambda}$. 
Proof. We can suppose that $\lambda<K$. Observe, that $\left(t_{n}^{*} t_{n}\right) R_{n}=R_{n}\left(t_{n}^{*} t_{n}\right)=0$. Hence $\left[t^{*} t\right]_{\mathcal{M}_{\mu}}[R]_{\mathcal{M}_{\mu}}=[R]_{\mathcal{M}_{\mu}}\left[t^{*} t\right]_{\mathcal{M}_{\mu}}=0$. Therefore by continuous functional calculus [20, Theorem VII. 1], for any continuous function $f$ on $[0, K]$ such that $f(0)=1$,

$$
f\left(\left[t^{*} t\right]_{\mathcal{M}_{\mu}}\right)[R]_{\mathcal{M}_{\mu}}=[R]_{\mathcal{M}_{\mu}} f\left(\left[t^{*} t\right]_{\mathcal{M}_{\mu}}\right)=[R]_{\mathcal{M}_{\mu}} .
$$

Now let $\left\{f_{n}\right\}_{n=1}^{\infty}$ be a monotonically decreasing sequence of continuous functions such that $f_{n}(0)=1$, and for any $x \in[0, K], \lim _{n \rightarrow \infty} f_{n}(x)=\chi_{[0, \lambda]}$. Then, $\left\{f_{n}\left(\left[t^{*} t\right]_{\mathcal{M}_{\mu}}\right)\right\}_{n=1}^{\infty}$ converges to $E_{\lambda}$ in the strong operator topology ([20, Theorem VII 2]), hence

$$
E_{\lambda}[R]_{\mathcal{M}_{\mu}}=[R]_{\mathcal{M}_{\mu}} E_{\lambda}=[R]_{\mathcal{M}_{\mu}}
$$

By the previous lemma,

$$
\operatorname{rk}_{1}\left([R]_{\mathcal{M}_{\mu}}\right)=\operatorname{tr}_{\mathcal{M}_{\mu}}\left([R]_{\mathcal{M}_{\mu}}\right) \leq \operatorname{tr}_{\mathcal{M}_{\mu}}\left(E_{\lambda}\right)
$$

Hence $\operatorname{rk}_{1}\left([t]_{\mathcal{M}_{\mu}}\right)=\operatorname{rk}_{1}\left(\left[t^{*} t\right]_{\mathcal{M}_{\mu}}\right) \leq 1-\operatorname{rk}_{1}\left([R]_{\mathcal{M}_{\mu}}\right)$, thus the proposition follows from (7).

Let $i_{\mu}: \mathbb{C} \Gamma \rightarrow \mathcal{M}_{\mu}$ be defined as $\rho \circ \psi$, where $\psi=\prod_{n=1}^{\infty} \psi_{n}$ as in Subsection 7.1 and

$$
\rho: B\left(\prod_{n=1}^{\infty} \operatorname{Mat}_{P_{n} \times P_{n}}(\mathbb{C})\right) \rightarrow \mathcal{M}_{\mu}
$$

be the quotient map from the bounded part of the direct product. The map $i_{\mu}$ is trace-preserving and extends to an injective trace-preserving map $\overline{i_{\mu}}: \mathcal{N}(\Gamma) \rightarrow \mathcal{M}_{\mu}$ (see e.g. [17, Proposition 7.1]. The map $f_{3}$ is just $i_{\mu}$ composed by the embedding $i_{\mathcal{M}}$ of $\mathcal{M}_{\mu}$ into its Ore localization. We prove that $f_{3}$ is rank-preserving later.

Now let us define the map $f_{6}$. Let $\tau$ be the map defined in Subsection 9.1. Then let $j=\rho \circ \tau: \mathcal{A}_{\phi} \rightarrow \mathcal{M}_{\mu}$ and let $s=i_{\mathcal{M}} \circ j$. Then $f_{6}$ is defined as the extension of $s$ to $\overline{\mathcal{A}_{\phi}}$. We need to show of course that $j$ is rank-preserving. By Lemma 9.3, we only need to show that

$$
\operatorname{rk}_{1}\left[\tau^{k}(\underline{a})\right]_{\mathcal{M}_{\mu}}=\operatorname{rk}_{2}\left[\tau^{k}(\underline{a})\right]_{\mathcal{M}_{\mu}^{a l g}},
$$

for any $\underline{a} \in \bigoplus_{A \in Z_{k}} \operatorname{Mat}_{A \times A}(\mathbb{C})$. However, this follows from Lemma 9.5 .

Now we prove $f_{3}=f_{6} \circ f_{4}$. This also shows that $f_{3}$ is rank-preserving. Again, it is enough to show that

$$
\lim _{k \rightarrow \infty}\left[\prod_{n=1}^{\infty}\left(\psi_{n}(a)-\psi_{n}^{k}(a)\right)\right]_{\mathcal{M}_{\mu}}=0 .
$$

We already proved that

$$
\lim _{k \rightarrow \infty}\left[\prod_{n=1}^{\infty}\left(\psi_{n}(a)-\psi_{n}^{k}(a)\right)\right]_{\mathcal{M}_{\mu}^{a l g}}=0 .
$$

Hence equation (9) follows from Proposition 9.1 
9.3. The third identity. We need to show that $f_{7} \circ f_{2}=f_{3}$. The map $f_{2}$ is defined as $i_{\mathcal{N}(\Gamma)} \circ i$, where $i: \mathbb{C} \Gamma \rightarrow \mathcal{N}(\Gamma)$ is the natural embedding, and $i_{\mathcal{N}(\Gamma)}$ is the embedding of $\mathcal{N}(\Gamma)$ into its Ore localization. The map $f_{7}: U(\mathcal{N}(\Gamma)) \rightarrow U\left(\mathcal{M}_{\mu}\right)$ is the extension of the injective map $\overline{i_{\mu}}$ to the Ore localizations. Note that $f_{7}$ is rank-preserving by [21, Lemma 2.2]. The map $f_{3}$ is defined above. By definition,

$$
\overline{i_{\mu}} \circ i=i_{\mu}
$$

Since $f_{7}$ is an extension of $\overline{i_{\mu}}$,

$$
f_{7} \circ i_{\mathcal{N}(\Gamma)}=i_{\mathcal{M}} \circ \overline{i_{\mu}}
$$

Therefore, $f_{7} \circ f_{2}=f_{3}$.

\section{REFERENCES}

[1] S. K. Berberian, Baer *-rings. Die Grundlehren der mathematischen Wissenschaften, Band 195. Springer-Verlag, New York-Berlin, (1972). MR.0429975 (55:2983)

[2] S. K. Berberian, The maximal ring of quotients of a finite von Neumann algebra. Rocky Mount. J. Math. 12 (1982) no. 1, 149-164. MR649748 (83i:16005)

[3] J. Dodziuk, P. Linnell, V. Mathai, T. Schick and S. Yates, Approximating $L^{2}$-invariants and the Atiyah conjecture. Dedicated to the memory of Jürgen K. Moser. Comm. Pure Appl. Math. 56 (2003), no. 7, 839-873. MR1990479 (2004g:58040)

[4] G. ElEK, The rank of finitely generated modules over group algebras. Proc. Amer. Math. Soc. 131 (2003), no. 11, 3477-3485. MR1991759 (2004i:43003)

[5] G. Elek and E. Szabó, Hyperlinearity, essentially free actions and $L^{2}$-invariants. The sofic property. Math. Ann. 332 (2005), no. 2, 421-441. MR2178069(2007i:43002)

[6] G. EleK, On the analytic zero divisor conjecture of Linnell. Bull. Lond. Math. Soc. 35 (2003), no. 2, 236-238. MR1952401 (2003m:20004)

[7] G. Elek And E. SzABó, Sofic groups and direct finiteness. Journal of Algebra 280 (2004), no. 2, 426-434. MR2089244 (2005d:16041)

[8] G. EleK, The strong approximation conjecture holds for amenable groups. Journal of Funct. Anal. 239 (2006), no. 1, 345-355. MR2258227 (2007m:43001)

[9] K. R. Goodearl, von Neumann regular rings. Robert E. Krieger Publishing Co., Inc., Malabar, FL, (1991). MR 1150975 (93m:16006)

[10] I. HALPERIN, Extensions of the rank function. Studia Math. 27 (1966), 325-335. MR0202773 $(34: 2633)$

[11] I. Kaplansky, Rings of operators. W. A. Benjamin, Inc., New York-Amsterdam (1968). MR.0244778 (39:6092)

[12] P. LinnelL, Embedding group algebras into finite von Neumann regular rings. Modules and Comodules, Trends in Mathematics, Birkhauser Verlag, 295-300. MR2742635

[13] P. Linnell And T. Schick, The Atiyah conjecture and Artinian rings. Pure and Applied Math. Quaterly 8 (2012), no. 2, 313-328.

[14] P. Linnell, W. Lück And R. Sauer, The limit of $F_{p}$-Betti numbers of a tower of finite covers with amenable fundamental groups. Proceedings of the AMS 139 (2011), no. 2, 421434. MR2736326

[15] W. LüCK, Approximating $L^{2}$-invariants by their finite-dimensional analogues. Geom. Funct. Anal. 4 (1994), no. 4, 455-481. MR.1280122 (95g:58234)

[16] D. S. Ornstein and B. Weiss, Entropy and isomorphism theorems for actions of amenable groups, J. Anal. Math 48 (1987), 1-141. MR.910005 (88j:28014)

[17] N. OzAWA, About the QWEP conjecture, International J. Math 15 (2004), 501-530. MR2072092(2005b:46124)

[18] V. G. Pestov, Hyperlinear and sofic groups: a brief guide. Bull. Symb. Logic 14 (2008), no. 4, 449-480. MR2460675 (2009k:20103)

[19] H. REICH, On the $K$ - and $L$-theory of the algebra of operators affiliated to a finite von Neumann algebra. K-theory 24 (2001), no. 4, 303-326. MR.1885124 (2003m:46103) 
[20] M. Reed And B. Simon, Methods of modern mathematical physics. I: Functional Analysis, Academic Press, New York (1980). MR751959 (85e:46002)

[21] A. Тном, Sofic groups and diophantine approximation. Comm. in Pure and Appl. Math., 61 (2008), no. 8, 1155-1171. MR2417890(2009j:46143)

The Alfred Renyi Institute of Mathematics, Hungarian Academy of Sciences, BuDAPEST, Hungary

E-mail address: elek@renyi.hu 